\title{
Unusual Case of Wilson's Disease and Coexisting Autoimmune Hepatitis
}

Iga Gromny*1, Monika Kukulska ${ }^{1}$, Elżbieta Poniewierka ${ }^{1}$ and Agnieszka Hałoń ${ }^{2}$

${ }^{1}$ Department of Gastroenterology and Hepatology, Poland

${ }^{2}$ Department of Pathomorphology and Oncological Cytology, Poland

Received: 阱 January 15, 2019; Published: 眥January 25, 2019

*Corresponding author: Iga Gromny, Department of Gastroenterology and Hepatology, Wrocław, Poland

\section{Opinion}

A 30-year-old woman was referred to the Department of Gastroenterology and Hepatology due to elevated transaminase levels. Her past medical history included Hashimoto's disease and was treated with levothyroxine. She reported no consumption of alcohol. The patient denied any gastrointestinal symptoms. Laboratory tests revealed increased level of serum aspartate aminotransferase and alanine aminotransferase with normal value of cholestatic parameters, albumin, and prothrombin time. Testing for viral hepatitis was negative. Hypergammaglobulinemia was noted and serum immunoglobulin G was elevated. Tests for antinuclear antibodies was positive. Liver biopsy showed piecemeal necrosis, interface hepatitis with lymphoplasmacytic infiltration and bridging fibrosis in nearly all portal tracts (Figures 1A-1D). Magnetic resonance imaging (MRI) of abdomen revealed enlarged liver with micronodular surface and regenerative nodules. Autoimmune hepatitis ( $\mathrm{AIH})$ was recognized and treatment with steroids was introduced which led to a decrease in liver enzymes. After three years of treatment patient noticed hand tremors. The diagnosis was extended to tests for Wilson's disease (WD).

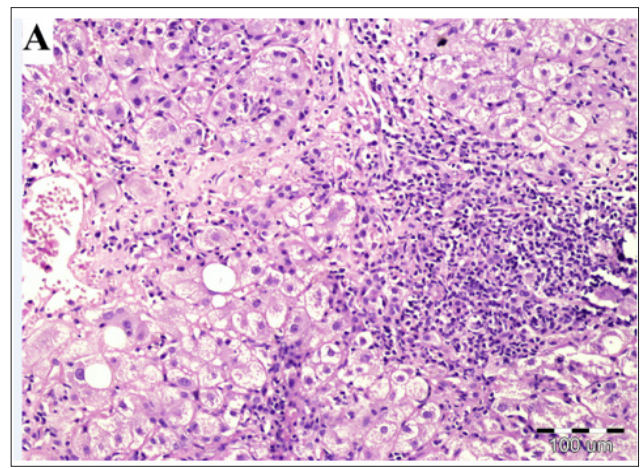

Figure 1A: Massive inflammatory infiltration and necrosis of hepatocytes.

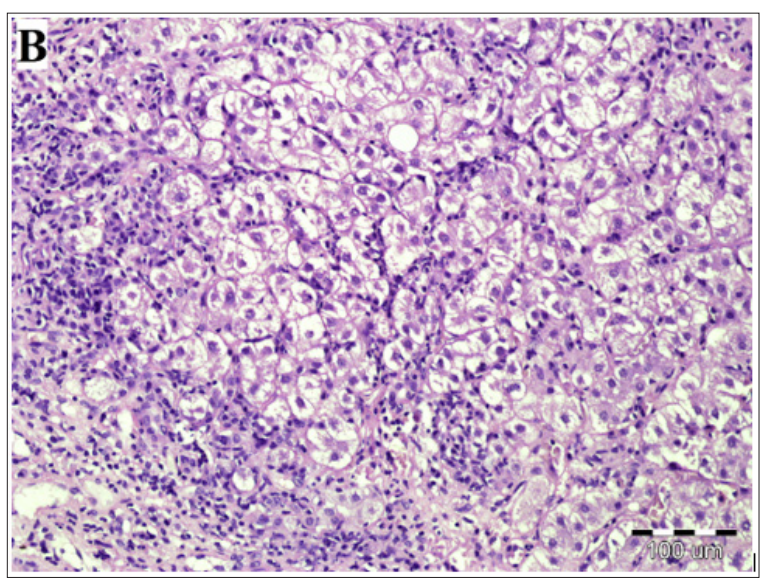

Figure 1B: lobular hepatitis and hepatic rosette formation.

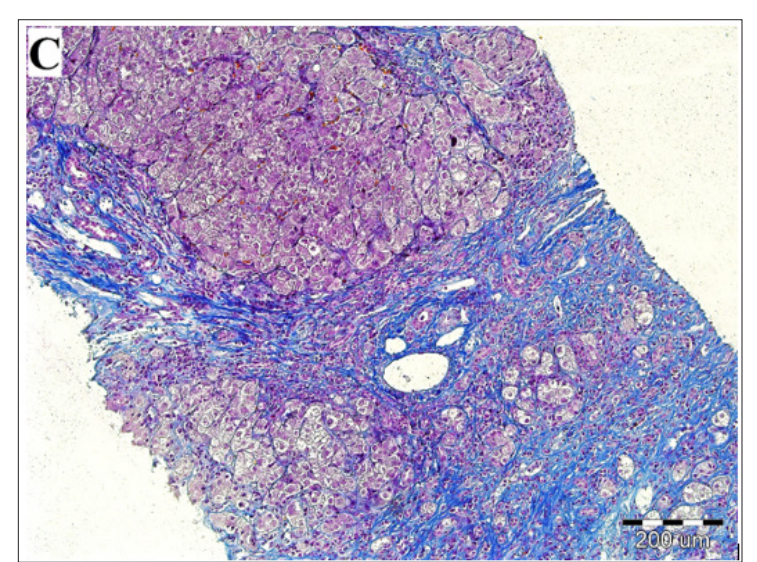

Figure 1C: Mallory's trichrome stain: liver parenchyma separated by fibrotic septa. 


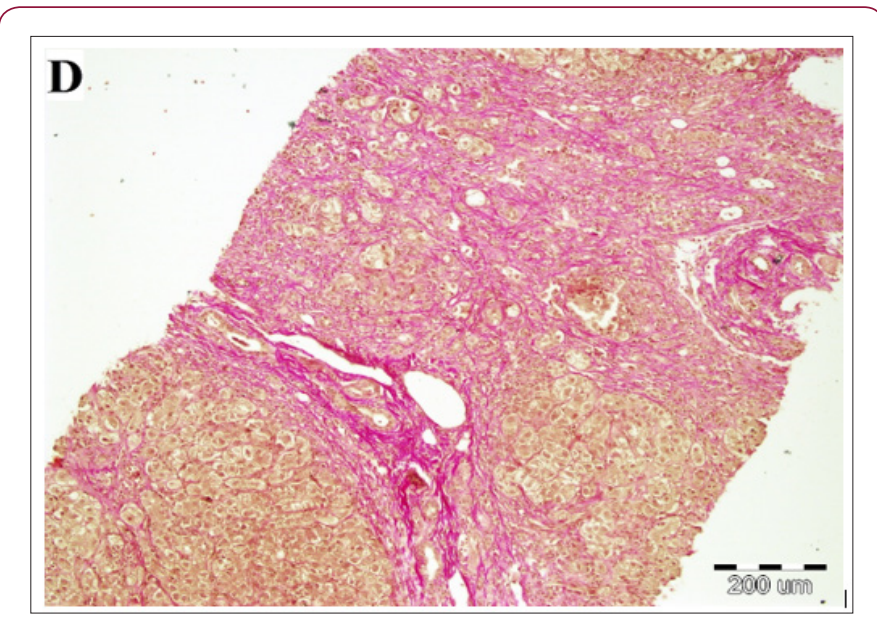

Figure 1D: Van Gieson's stain- massive fibrosis.

The level of serum ceruloplasmine was decreased at simultaneously normal level of 24 hours urinary copper. An ophthalmologist confirmed Kayser Fleischer rings. Genetic testing was performed and ATP7B mutation was found. Head MRI showed the hyperintensity in bilateral basal ganglia. Basic on conducted tests WD was diagnosed and penicillamine was introduced into the treatment. The coexistence of AIH and WD is rarely reported. Milkiewicz at all described two such cases. Patients were initially diagnosed as AIH. The diagnosis of WD was made after a few years. Diagnosis has been extended because of an ineffective steroid therapy and persistent elevated liver function tests [1]. Because of a delayed WD diagnosis in some cases liver transplantation is necessary [2]. AIH is a progressive liver disease with unknown etiology which mainly affects women.

The diagnosis can be difficult and is based on autoimmune serology, histological features on liver biopsy and a favorable response to immunosuppressive therapy. The exclusion of toxic, viral, metabolic and genetic causes of chronic or acute liver disease is necessary. The disease can be asymptomatic, with unspecific symptoms or recognized as even fulminant hepatitis [3,4]. WD is an autosomal recessive condition caused by a mutation in the ATP7B gene. It is characterized by excessive copper deposition mainly in liver (hepatic variant) and brain

(neuropsychiatric variant), but also in other organs such as cornea and kidney [5]. There is no completely reliable test for WD, but levels of ceruloplasmine and copper in the blood, as well as copper excreted in urine during a 24-hour period, are useful for the diagnosis [3]. It is important to exclude the presence of Kayser Fleischer rings in cornea during ophthalmological examination [4]. Liver biopsy for histology and histochemistry and copper quantification is recommended. Genetic testing may be used to screen the family members of those affected. The basic therapy for WD is copper chelation therapy with penicillamine and trientine [5]. Our conclusion is that extended screening is necessary despite of AIH diagnosis, especially when response for appropriate treatment is poor.

\section{References}

1. Gatselis NK, Zachou K, Koukoulis GK, Dalekos GN (2015) Autoimmune hepatitis, one disease with many faces: etiopathogenetic clinicolaboratory and histological characteristics. World J Gastroenterol 21(1): 60-83.

2. Liwinski T, Schramm C (2017) Autoimmune hepatitis-update on clinical management in 2017. Clin Res Hepatol Gastroenterol 41(6): 617-625.

3. J Hepatol (2012) Liver EA for the S of the. EASL Clinical Practice Guidelines: Wilson's disease 56: 671-685.

4. Milkiewicz P, Saksena S, Hubscher SG, Elias E (2000) Wilson's disease with superimposed autoimmune features: Report of two cases and review. J Gastroenterol Hepatol 15(5): 570-574.

5. Santos RG, Alissa F, Reyes J, Teot L, Ameen N, et al. (2005) Fulminant hepatic failure: Wilson's disease or autoimmune hepatitis? Implications for transplantation. Pediatr Transplant 9(1): 112-116.
ISSN: 2574-1241

DOI: 10.26717/BJSTR.2019.13.002432

Iga Gromny. Biomed J Sci \& Tech Res

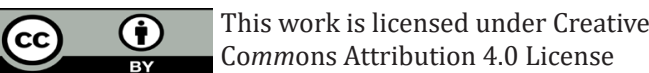

Submission Link: https://biomedres.us/submit-manuscript.php

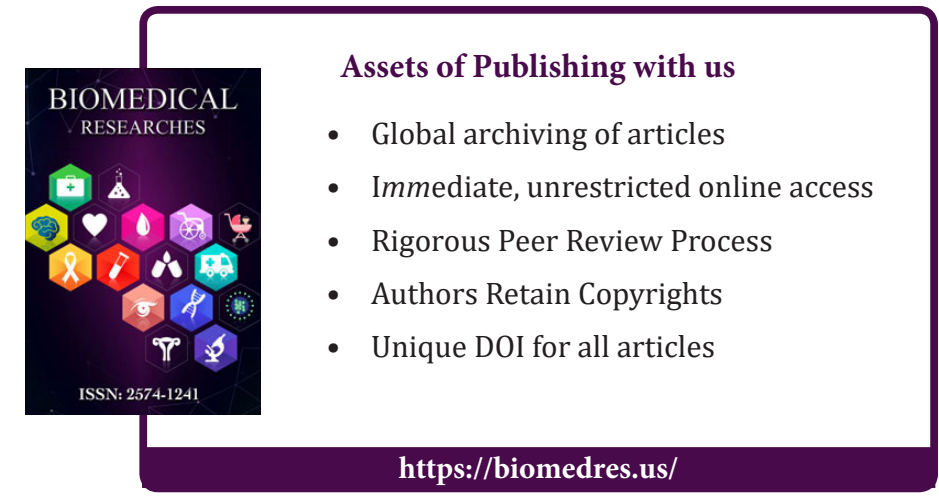

